\title{
Daily tea drinking is not associated with newly diagnosed non-alcoholic fatty liver disease in Chinese adults: the Tianjin chronic low-grade systemic inflammation and health cohort study
}

Yang Xia ${ }^{1+}$, Xuena Wang ${ }^{2+}$, Shunmin Zhang ${ }^{2}$, Qing Zhang ${ }^{3}$, Li Liư ${ }^{3}$, Ge Meng ${ }^{2}$, Hongmei Wu ${ }^{2}$, Xue Bao ${ }^{2}$, Yeqing $\mathrm{Gu}^{2}$, Shaomei Sun ${ }^{3}$, Xing Wang ${ }^{3}$, Ming Zhou ${ }^{3}$, Qiyu Jia ${ }^{3}$, Kun Song ${ }^{3}$, Qijun Wu ${ }^{1}$, Kaijun $\mathrm{Niu}^{2,3^{*}}$ and Yuhong Zhao ${ }^{1 *}$ (D)

\begin{abstract}
Background: Previous studies have reported that tea extract supplementation has potential benefits on the risk factors of non-alcoholic fatty liver disease (NAFLD); however, no study has investigated the direct effect of daily tea consumption on the prevalence of NAFLD in the general population. This cross-sectional study aimed to evaluate the associations between tea consumption and the prevalence of newly diagnosed NAFLD among Chinese adults.

Methods: The present cross-sectional study was based on the Tianjin Chronic Low-grade Systemic Inflammation and Health Cohort Study. In total, 19,350 participants were enrolled in the analyses. Tea consumption was assessed via a self-administered food frequency questionnaire. NAFLD was diagnosed via liver ultrasonography and no history of heavy alcohol intake. Logistic regression analysis was used to estimate the associations between tea consumption and the prevalence of NAFLD.
\end{abstract}

Results: Consumption of green tea, oolong tea, and black tea were positively associated with the prevalence of newly diagnosed NAFLD before adjustments. Compared with the participants who never drink tea, the odds ratios ( $95 \%$ confidence interval) of newly diagnosed NAFLD in the highest categories ( $\geq 1$ cup/day) of green tea, oolong tea, black tea, and jasmine tea were $1.48(1.33,1.65), 1.50(1.33,1.68)$, and $1.28(1.13,1.46)$, and $1.36(1.20,1.54)$ before adjustments, respectively. However, no significant association was found between tea consumption and the prevalence of NAFLD after adjusting for socio-demographic, behavioural, anthropometric, dietary, and clinical confounding factors.

Conclusion: There is no significant association between daily tea drinking and newly-diagnosed NAFLD in general Chinese adults.

Keywords: Tea consumption, Non-alcoholic fatty liver disease, Prevalence

\footnotetext{
* Correspondence: nkj0809@gmail.com; niukaijun@tmu.edu.cn; zhaoyuhong@sj-hospital.org

${ }^{\dagger}$ Yang Xia and Xuena Wang contributed equally to this work.

${ }^{2}$ Nutritional Epidemiology Institute and School of Public Health, Tianjin Medical University, 22 Qixiangtai Road, Heping District, Tianjin 300070, Tianjin, China

'Department of Clinical Epidemiology, Shengjing Hospital of China Medical

University, No. 36, San Hao Street, Shenyang 110004, Liaoning, China

Full list of author information is available at the end of the article
}

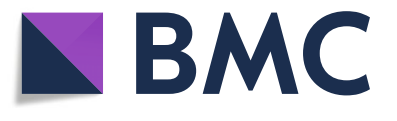

(c) The Author(s). 2019 Open Access This article is distributed under the terms of the Creative Commons Attribution 4.0 International License (http://creativecommons.org/licenses/by/4.0/), which permits unrestricted use, distribution, and reproduction in any medium, provided you give appropriate credit to the original author(s) and the source, provide a link to the Creative Commons license, and indicate if changes were made. The Creative Commons Public Domain Dedication waiver (http://creativecommons.org/publicdomain/zero/1.0/) applies to the data made available in this article, unless otherwise stated. 


\section{Introduction}

Non-alcoholic fatty liver disease (NAFLD) is rapidly becoming the most common chronic liver disease and potentially affects $25 \%$ of the global adult population [1]. NAFLD develops without alcohol abuse and represents a spectrum of liver disease encompassing simple fatty infiltration, fat and inflammation, and cirrhosis [2]. Moreover, NAFLD is associated with metabolic syndrome [3], type 2 diabetes mellitus [4], and chronic kidney disease [5]. However, there is a lack of current evidence regarding the efficacy and safety profiles of pharmacotherapies aimed at treating NAFLD. Lifestyle management, including sustained weight loss, healthy diet, and increased physical activity (PA), is still an important approach to treating NAFLD $[6,7]$. Thus, it is important to identify modifiable risk factors and develop preventive strategies to decrease the disease burden of NAFLD.

Tea (Camellia sinensis Theaceae) was discovered in China in $3000 \mathrm{BC}$ or earlier [8]. Tea in its various processed forms (i.e., non-fermented green tea, partlyfermented oolong tea, and fermented black tea) represents the most widely consumed beverage worldwide [9]. In Asia, tea has been regarded to process significant healthpromoting effects for centuries [10]. Most recently, a meta-analysis that included four randomized controlled trials suggested that there are potential benefits of green tea supplementation on the risk factors of NAFLD [11]. All the studies pooled in that meta-analysis explored the effect of tea consumption on the risk factors of NAFLD by using green tea extract supplementation as treatment [11]. However, daily tea consumption, different from tea extract supplementation, contains lower amounts of tea polyphenol. To the best of our knowledge, no study has explored the associations between daily tea consumption and the prevalence of NAFLD. Thus, we designed this crosssectional study of the general population to explore the associations between daily tea consumption and the prevalence of newly diagnosed NAFLD among Chinese adults.

\section{Materials and methods \\ Participants}

This cross-sectional study was based on the Tianjin Chronic Low-grade Systemic Inflammation and Health (TCLSIHealth) Cohort Study, which is a large prospective dynamic cohort study that focuses on the relationships between chronic low-grade systemic inflammation and the health status of a population living in Tianjin, China [12]. Participants were recruited during their annual health examinations at the Tianjin Medical University General Hospital-Health Management Center and community management centres in Tianjin. This dynamic cohort study was launched in 2007. Furthermore, a detailed lifestyle questionnaire had been administered to about 70-
$80 \%$ of randomly selected subjects from this population since May 2013. The questionnaire covered questions about family income, marital status, employment status, educational level, physical activity, sleep habits, dietary habits, overall computer/mobile device usage time, television time, history of prior infections, and use of medicines as well as physical performance tests.

A total of 32,165 participants without acute inflammatory disease completed a comprehensive health examination and the study questionnaire. We excluded participants who changed their lifestyles (including dietary intake, drinking, smoking, physical activity, and sleeping habits) (assessed via a question in the questionnaire) in the last five years $(n=$ $7364)$, or those had a history of cardiovascular disease $(n=$ $1057)$, cancer $(n=173)$, NAFLD $(n=3344)$, alcoholic fatty liver disease $(n=775)$, and other live diseases (including chronic hepatitis $\mathrm{B}$ or $\mathrm{C}$, autoimmune liver disease, and liver surgery, $n=102$ ). The final study population comprised 19,350 individuals.

\section{Assessment of tea consumption}

Dietary intake was assessed using a validated food frequency questionnaire (FFQ) that included 100 food items with specified serving sizes. The FFQ included seven frequency categories ranging from 'almost never eat' to 'twice or more per day' for foods and eight frequency categories ranging from 'almost never drink' to 'four or more times per day' for beverages. The reproducibility and validity of the questionnaire have been assessed in a random sample of 150 participants living in Tianjin by comparing the data from the questionnaire with the data from two dietary questionnaires collected approximately three months apart and four-day weighed dietary records (WDRs). Spearman's rank correlation coefficient for energy intake between two food frequency questionnaires administered three months apart was 0.68. The correlation coefficients for food items (fruits, vegetables, fish, meat, and beverages) between two food frequency questionnaires administered three months apart ranged from 0.62 to 0.79 . Spearman's rank correlation coefficient for energy intake as assessed via the WDRs and FFQ was 0.49. Correlation coefficients for nutrients (vitamin $\mathrm{C}$, vitamin $\mathrm{E}$, polyunsaturated fats, saturated fats, carbohydrates and calcium) as assessed via the WDRs and FFQ ranged from 0.35 to 0.54 .

The mean daily intake of nutrients was calculated using an ad hoc computer program developed to analyse the questionnaire. The Chinese food composition tables [13] were used as the nutrient database. Factor analysis was applied to generate the major dietary patterns and food loading on all 100 food items and beverages (gram). Factors were named descriptively according to the food items that showed high loading (absolute value $>0.3$ ) with respect to each dietary pattern as follows: sweet 
foods pattern, vegetable pattern and animal foods pattern (Additional file 1: Table S1). The scores of dietary patterns were used for further analyses as confounding factors.

The participants indicated the frequency of consumption of green tea, oolong tea, black tea, and jasmine tea over the previous one month in terms of the specified serving size by selecting one of the following eight frequency categories: almost never, < 1 cup/week, 1 cup/ week, 2-3 cups/week, 4-6 cups/week, 1 cup/day, 2-3 cups/day, and $\geq 4$ cups/day ( $200 \mathrm{ml}$ per cup). In the study region, a cup of tea has a typical volume of $200 \mathrm{ml}$. In the present study, the frequency of intake of green tea, oolong tea, and black tea were categorised as: almost never, $<1$ cup/week, $1-6$ cups/week and $\geq 1$ cup/day.

\section{Liver ultrasonography and definitions of NAFLD}

Liver ultrasonography was performed by trained sonographers using a TOSHIBA SSA-660A ultrasound machine (Toshiba, Tokyo, Japan) with a $2-5-\mathrm{MHz}$ curved array probe. According to the revised definition and treatment guidelines for NAFLD published by the Chinese Association for the Study of Liver Disease in 2010 [14], we defined 'heavy drinking' as an intake of $>140 \mathrm{~g}$ of alcohol per week among men and $>70 \mathrm{~g}$ per week among women. Total alcohol intake in the preceding week was assessed using the FFQ. Participants were diagnosed as having NAFLD if they had evidence on abdominal ultrasonography (brightness of liver and a diffusely echogenic change in the liver parenchyma) and no history of heavy drinking.

\section{Assessment of other variables}

The educational level was assessed by asking the question 'what is the highest degree you earned?' with two possible response categories: <college graduate or $\geq$ college graduate. Employment status was classified as either senior officials and managers or professionals. Information on smoking ('never,' 'former,' and 'current smoking') and drinking ('never,' 'former,' 'current drinking everyday', and 'current drinking sometimes') status of the participants was obtained via a questionnaire survey. PA in the most recent week was assessed using the short form of the International Physical Activity Questionnaire [15]. The questionnaire asked whether subjects had performed any activities from the following categories during the preceding week: walking, moderate activity (household activity or child care), and vigorous activity (running, swimming, or other sporting activities). Metabolic equivalents (METs; hours per week) were calculated using corresponding MET coefficients (3.3, 4.0, and 8.0, respectively) according to the following formula: MET coefficient of activity $x$ duration (hours) $\times$ frequency (days). Total PA levels were assessed by combining separate scores for different activities. Weight and height were assessed using an automatic height and weight measurement instrument (Omron HNH-219; Omron, Kyoto, Japan) with a standard protocol. Body mass index (BMI) was calculated by dividing the weight in kilograms by the square of the height in meters $\left(\mathrm{kg} / \mathrm{m}^{2}\right)$. Fasting blood samples were obtained via venipuncture of the cubital vein and immediately mixed with EDTA. Waist circumference was measured at the umbilical level with participants standing and breathing normally. Blood pressure was measured twice from the upper left arm using a TM-2655P automatic device (A\&D Co., Tokyo, Japan) after five minutes of rest in a seated position. The mean of these two measurements was taken as the blood pressure value. Hypertension was defined as average systolic $\mathrm{BP} \geq 140 \mathrm{mmHg}$ or average diastolic BP $\geq 90 \mathrm{mmHg}$ or use of antihypertension medications [16]. Blood samples for the analysis of fasting blood glucose and lipid levels were collected in siliconised vacuum plastic tubes. Fasting blood sugar level was measured via the glucose oxidase method, triglyceride level was measured via enzymatic methods, and high-density lipoprotein cholesterol level was measured via the chemical precipitation method using reagents obtained from Roche Diagnostics on an automatic biochemistry analyser (Roche Cobas 8000 modular analyser; Roche, Mannheim, Germany). HbA1c separation and quantification were conducted by a high-performance liquid chromatography analyzer (HLC723 G8; Tosoh, Tokyo, Japan). In addition, an oral glucose tolerance test was performed, and postprandial glucose was determined in blood samples obtained $2 \mathrm{~h}$ after oral administration of a standard $75 \mathrm{~g}$ glucose solution. Type 2 diabetes was defined as having fasting blood glucose $\geq 7.0$ $\mathrm{mmol} /$, or 2 -h postprandial blood glucose $\geq 11.1 \mathrm{mmol} / \mathrm{l}$, or $\mathrm{HbA} 1 \mathrm{c} \geq 6.5 \%(48 \mathrm{mmol} / \mathrm{mol})$, or a history of diabetes based on the 2014 American Diabetes Association criteria [17]. Hyperlipidemia was defined as TC $\geq 5.20 \mathrm{mmol} / \mathrm{L}$, or $\mathrm{TG} \geq 1.70 \mathrm{mmol} / \mathrm{L}$, or self-reported clinically diagnosed hyperlipidemia according to 2016 Chinese guidelines for the management of dyslipidemia in adults [18].

\section{Statistical analysis}

In the assessment of characteristics of participants according to NAFLD status, continuous variables have been presented as the least square mean (with 95\% confidence interval, $[\mathrm{CI}]$ ) and examined using analysis of variance. Categorical variables have been presented as percentages and examined using chi-square test. Associations between tea drinking and the prevalence of newly diagnosed NAFLD were assessed via conditional logistic regression analysis. Odds ratios (ORs) and 95\% CIs were calculated. All $P$ values for linear trend were calculated according to the categories of tea consumption (almost never: $1,<1$ cup/week: 2, 1-6 cups/week: $3, \geq 1$ cup/day: 4) based on logistic regression. Model 1 was adjusted for 
age, BMI, and sex. Model 2 was additionally adjusted for energy intake $(\mathrm{kJ} / \mathrm{d})$, type 2 diabetes, hypertension, hyperlipidemia, physical activity, educational level, household income, smoking status, drinking status, employment status, family history of cardiovascular disease, cancer, and diabetes, intake of sweet foods pattern, vegetable pattern and animal foods pattern, and consumption of two other kinds of tea. All analyses were performed using the Statistical Analysis System 9.3 edition for Windows (SAS Institute Inc., Cary, NC, USA) and STATA (version 12.1; Stata Corp LP, College Station, TX, USA). All $P$-values were two-tailed and differences with $P<0.05$ were considered statistically significant.

\section{Results}

\section{Characteristics of participants}

Table 1 shows the age- and sex-adjusted characteristics according to NAFLD status. Among the 19,350 participants, $19.4 \%$ were classified as newly diagnosed NAFLD. After adjustments for age and sex where appropriate, participants with NAFLD tended to be men, older, of lower education level, less likely to be employed as managers $(P<0.0001)$, and current or ex-smokers, who drank sometimes, had lower daily energy intake and lower intake of vegetable pattern $(P<0.0001)$, had a higher prevalence of type 2 diabetes, hypertension, hyperlipidaemia, and family history of diabetes, and higher BMI and alanine aminotransferase level $(P<0.0001)$.

\section{Tea consumption and NAFLD}

The associations between tea consumption and the prevalence of newly diagnosed NAFLD are shown in Table 2. Compared with the participants who never drank tea, the ORs (95\% CI) of newly diagnosed NAFLD in the highest category ( $\geq 1 \mathrm{cup} /$ day) of green tea, oolong tea, black tea, and jasmine tea before adjustments were1.48 $(1.33,1.65)$, 1.50 (1.33, 1.68), $1.28(1.13,1.46)$, and $1.36(1.20,1.54)$, respectively. However, no significant association was found between tea consumption and NAFLD after adjustment for socio-demographic, behavioural, anthropometric, dietary, and clinical confounding factors.

\section{Discussion}

The present study, to the best of our knowledge, is the first to explore the associations between daily tea consumption and the prevalence of newly-diagnosed NAFLD in a general population with a large sample size. Sociodemographic, behavioural, metabolic, and health status confounding factors were adjusted in logistic regression to explore the associations between daily tea consumption and the prevalence of NAFLD. Furthermore, we also adjusted scores of major dietary patterns in the study population in case the associations between daily tea consumption and the prevalence of NAFLD may be affected by intake of dietary confounding factors (i.e. high consumption of red meat [19], fruits [20], sweet products, desserts, and sugar [21]). No significant association was detected between daily consumption of green tea, oolong tea, black tea, and jasmine tea and the prevalence of newly diagnosed NAFLD in Chinese adults after adjustments.

Previous studies have focused on the effect of tea extract on NAFLD. A previous meta-analysis of four clinical trials suggested that green tea extract supplementation yield benefits on NAFLD related risk factors [11]. The results indicated green tea extract supplementation significantly altered the blood levels of alanine and aspartate aminotransferases [11]. Meanwhile, green tea extract supplementation was found to yield a favourable effect on BMI, triacylglycerol, total cholesterol, and low-density lipoprotein cholesterol [11]. Previous studies also suggested that green tea extract could improve obesity, hyperlipidaemia, and NAFLD in mice [22]. The mechanisms underlying how green tea protects against NAFLD are mostly related to catechins present in tea. The evidence from in vitro and in vivo studies indicates that green tea inhibits dietary lipid absorption [23], decreases lipid accumulation in the liver and adipose tissue [24], improves insulin sensitivity [25], and exerts antioxidant properties [26].

However, no study has reported on the beneficial effect of daily tea consumption and the prevalence of NAFLD. We found a positive association between consumption of tea and the prevalence of newly-diagnosed NAFLD before adjustments. This may be due to the fact that participants who drank tea tended to be men and older. Meanwhile, men and older have higher prevalence of NAFLD than women and younger participants [27]. There was no association between tea consumption and NAFLD after adjustment of confounding factors. The results are not in line with those of previous studies which found that tea extracts are associated with a lower prevalence of the risk factors of NAFLD. Daily tea consumption yields a lower content of catechins than in tea extract. A previous meta-analysis showed that catechin intake is associated with a lower prevalence of NAFLD risk factors [11], and that the daily intake amounts of catechin in the treatment groups of previous randomised clinical trials were $>500 \mathrm{mg}$. However, the amounts of catechins in most commercially available green teas is about $60 \mathrm{mg}$ per $200 \mathrm{ml}$ [28], which is equal to one cup in the present study. Thus, the low amount of catechins consumed daily in tea may not be enough to have a beneficial effect on NAFLD. Moreover, a previous study conducted among Chinese women showed that tea consumption is associated with the serum concentration of total organochlorine pesticides $(\mathrm{r}=0.14, P<0.05)$ [29]. A previous study demonstrated that green tea consumption was positively associated with the risk of type 2 
Table 1 Age- and sex- adjusted characteristics according to NAFLD status ${ }^{\text {a }}$

\begin{tabular}{|c|c|c|c|}
\hline \multirow[t]{2}{*}{ Characteristics } & \multicolumn{2}{|l|}{ NAFLD status } & \multirow{2}{*}{$\begin{array}{l}P \text { value } \\
\mathrm{b}\end{array}$} \\
\hline & No $(n=15,589)$ & Yes $(n=3761)$ & \\
\hline Sex (male \%) & 46.6 & 81.6 & $<0.0001$ \\
\hline Age $(y)$ & $37.5(37.4,37.7)^{a}$ & $41.3(41.0,41.7)$ & $<0.0001$ \\
\hline BMl & $22.9(22.8,23.0)$ & $27.2(27.1,27.3)$ & $<0.0001$ \\
\hline Physical activity (Mets × hours/week) & $10.5(10.2,10.7)$ & $10.2(9.8,10.6)$ & 0.31 \\
\hline Energy intake (kJ/d) & $8457.9(8420.3,8496.0)$ & $8257.0(8180.4,8334.9)$ & $<0.0001$ \\
\hline Education ( $\geq$ college graduate, \%) & 71.2 & 59.9 & $<0.0001$ \\
\hline Household income ( $\geq 10,000$ Yuan, \%) & 33.4 & 31.6 & 0.04 \\
\hline $\operatorname{ALT}(U / L)$ & $15.3(15.2,15.4)$ & $25.0(24.6,25.4)$ & $<0.0001$ \\
\hline Type 2 diabetes (\%) & 1.3 & 8.4 & $<0.0001$ \\
\hline Hypertension (\%) & 12.7 & 40.3 & $<0.0001$ \\
\hline Hyperlipidaemia (\%) & 31.1 & 66.3 & $<0.0001$ \\
\hline \multicolumn{4}{|l|}{ Dietary pattern score (multiply by 100 ) } \\
\hline Sweets foods pattern & $0.3(-1.3,1.9)$ & $-2.4(-5.7,0.9)$ & 0.15 \\
\hline Vegetable pattern & $2.2(0.7,3.8)$ & $-6.4(-9.6,-3.1)$ & $<0.0001$ \\
\hline Animal foods pattern & $1.7(0.2,3.3)$ & $-2.1(-5.3,1.1)$ & 0.04 \\
\hline \multicolumn{4}{|l|}{ Smoking status (\%) } \\
\hline Smoker & 14.0 & 27.6 & $<0.0001$ \\
\hline Ex-smoker & 3.5 & 6.2 & $<0.0001$ \\
\hline Non-smoker & 82.5 & 66.2 & $<0.0001$ \\
\hline \multicolumn{4}{|l|}{ Drinker (\%) } \\
\hline Everyday & 3.6 & 2.1 & $<0.0001$ \\
\hline Sometime & 52.6 & 61.7 & $<0.0001$ \\
\hline Ex-drinker & 10.3 & 12.4 & $<0.001$ \\
\hline Non-drinker & 33.4 & 23.7 & $<0.0001$ \\
\hline \multicolumn{4}{|l|}{ Employment status (\%) } \\
\hline Managers & 44.6 & 39.7 & $<0.0001$ \\
\hline Professionals & 16.0 & 16.7 & 0.29 \\
\hline Other & 39.5 & 43.6 & $<0.0001$ \\
\hline \multicolumn{4}{|l|}{ Family history of diseases (\%) } \\
\hline CVD & 26.0 & 22.4 & $<0.0001$ \\
\hline Hypertension & 46.6 & 47.3 & 0.44 \\
\hline Diabetes & 23.1 & 27.7 & $<0.0001$ \\
\hline
\end{tabular}

${ }^{a}$ NAFLD, non-alcoholic fatty liver disease; CVD, cardiovascular disease; BMI, body mass index; ALT, alanine aminotransferase

${ }^{b}$ Analysis of variance or chi-square test with adjustments for age and sex where is appropriate

c Least square mean (95\% confidence interval) (all such values)

diabetes in Chinese adults (hazard ratio: 1.20; $95 \% \mathrm{CI}$ : $1.14,1.27$ ) [30]. Liu et al. suggested that pesticide contamination of tea could underlie the association between daily tea consumption and the incidence of type 2 diabetes [30]. Significant increases in serum biochemical indices, including the levels of alanine aminotransferases, aspartate aminotransferase, alkaline phosphatase, total cholesterol, triglycerides, low-density lipoprotein cholesterol, and lipid peroxidation by-products have been observed experimentally after subchronic or chronic exposure to organophosphates [31, 32]. Thus, it is possible that the pesticide residue in tea, released into hot water could attenuate the beneficial effect of tea in the present study. Further studies are warranted to directly assess pesticide levels in tea and confirm the findings of the present study.

It is also possible that the participants with NAFLD have changed their habit of tea consumption to achieve healthy lifestyles. Thus, the reverse causation may partly explain the findings of the present study. However, we 
Table 2 Association between tea consumption and NAFLD *

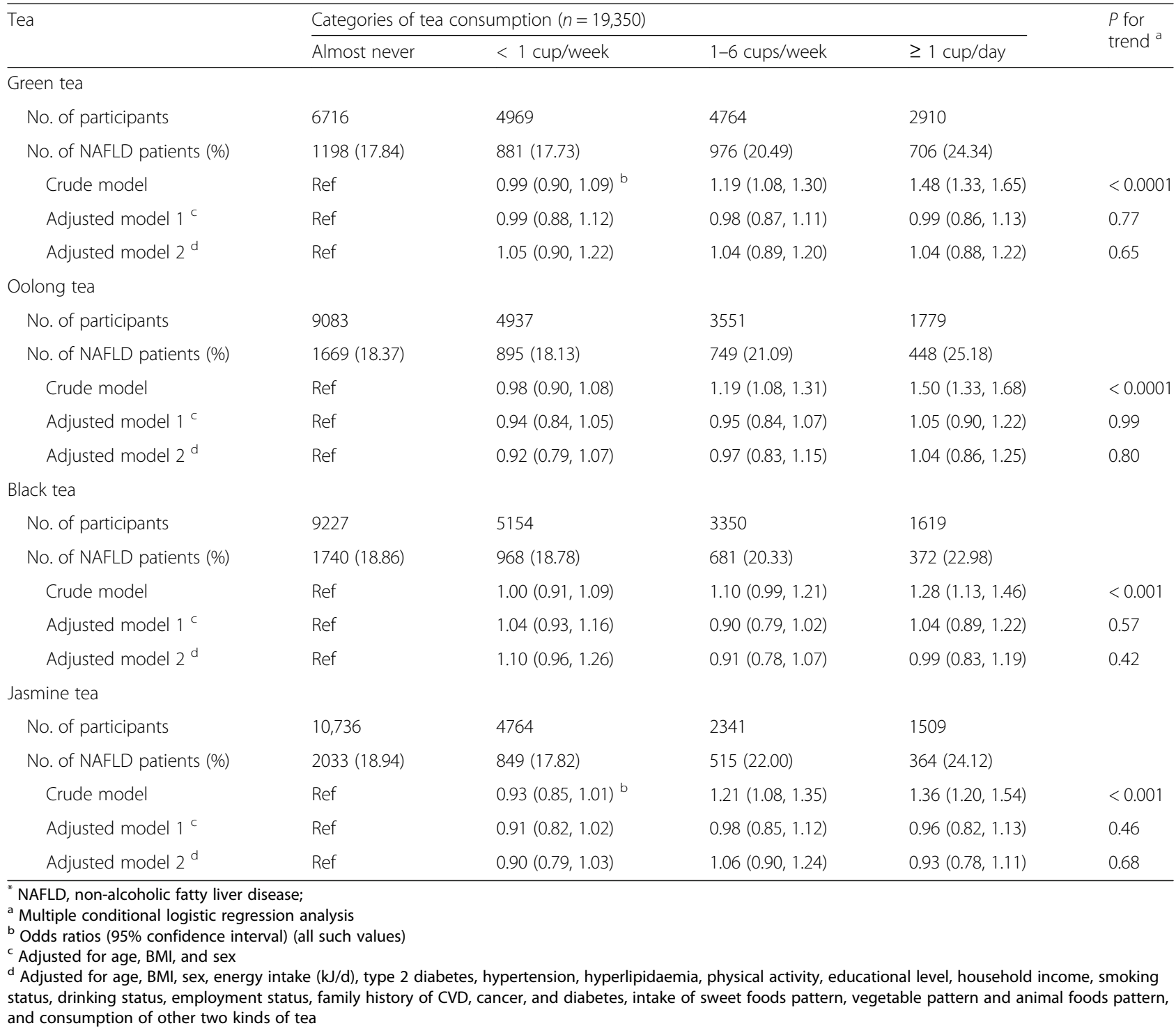

excluded participants who had changed their lifestyles (including dietary intake, drinking, smoking, activity, and sleeping) in the preceding five years. Moreover, we also excluded participants who had a history of NAFLD. The participants with NAFLD in the present study are newly diagnosed and have not changed their habits of tea consumption in the preceding five years. Thus, reverse causation was corrected as much as possible. Another strength of the present study is that although a previous study indicated that tea extract supplementation might yield beneficial effects in patients with NAFLD [11], there is limited evidence for the relationship between daily tea consumption and the prevalence of NAFLD in the general population. Although the underlying mechanism remains unclear, the present study extended the evidence in this regard, and found no association between daily tea consumption and the prevalence of newly diagnosed NAFLD among Chinese adults.

The present study has some limitations. First, due to the nature of the self-reporting questionnaire, recall bias existed and the amount of tea consumed may not have been precisely reported. Second, we excluded participants with health conditions; thus, the final sample may not be representative of the population. However, it is reasonable to exclude participants with cardiovascular disease and cancer when estimating the associations between tea consumption and NAFLD. Third, although we considered as many covariates as possible, we cannot rule out the possibility that unmeasured factors might contribute to the associations observed. Fourth, all the previous studies on tea and NAFLD showed effects at dosage higher than $500 \mathrm{mg}$ of catechins/day (equivalent 
to 9 cups of green tea). It is possible that the lack of association is due to the fact that the proportion of participants who drink enough cups of tea per day is too low in a general population. Further randomized controlled trials are needed to explore the associations between excessive tea drinking and the prevalence of NAFLD. Fifth, the validity of the short form of IPAQ has not been tested in the study population and the physical activity may not be exact. Finally, liver biopsy, the gold standard for the diagnosis of liver disease, was not performed in the present study, due to the apparently healthy study population. Instead, we diagnosed fatty liver via hepatic ultrasonography. This technique has a sensitivity of $89 \%$ and a specificity of $93 \%$ and is widely used in population-based studies due to its noninvasiveness and easy accessibility [33].

\section{Conclusions}

Despite the limitations, the results of the present study demonstrate that consumption of tea ( $\geq 1$ cup/day) is not associated with the prevalence of newly diagnosed NAFLD among general Chinese adults. Further studies are needed to explore the associations between more cups of tea drinking (e.g., equivalent to $500 \mathrm{mg}$ of catechins/day) and the prevalence of NAFLD.

\section{Supplementary information}

Supplementary information accompanies this paper at https://doi.org/10. 1186/s12937-019-0502-y.

Additional file 1: Table S1. The factor loadings scores of primary food groups of dietary patterns.

\section{Acknowledgements}

We gratefully thank all the participants in the study and Tianjin Medical University General Hospital-Health Management Center for the opportunity to perform the study.

\section{Authors' contributions}

Y. X., K. N., and Y, Z., designed research; Y. X., X. W., S. Z., Q. Z., L. L., G. M., H. W., X. B., Y. G., S. S., X. W., M. Z., Q. J. and K. S., conducted research; Y. X. analyzed data; $Y$. X. and Q. W. wrote the paper. K. N. and $Y, Z$. had primary responsibility for final content. All authors read and approved the final manuscript.

\section{Funding}

This study was supported by grants from the National Natural Science Foundation of China (No. 81903302, 91746205, and 81673166), China; China Postdoctoral Science Foundation (No. 2018 M641753).

\section{Availability of data and materials}

The datasets generated and/or analysed during the current study are not publicly available due to that it is an ongoing cohort study but are available from the corresponding author on reasonable request.

\section{Ethics approval and consent to participate}

The protocol of this study was approved by the Institutional Review Board of the Tianjin Medical University and participants gave written informed consent before participation in the study.
Consent for publication

Not applicable.

\section{Competing interests}

The authors declare that they have no competing interests.

\section{Author details}

${ }^{1}$ Department of Clinical Epidemiology, Shengjing Hospital of China Medical University, No. 36, San Hao Street, Shenyang 110004, Liaoning, China. ${ }^{2}$ Nutritional Epidemiology Institute and School of Public Health, Tianjin Medical University, 22 Qixiangtai Road, Heping District, Tianjin 300070, Tianjin, China. ${ }^{3}$ Health Management Centre, Tianjin Medical University General Hospital, Tianjin, China.

Received: 10 September 2019 Accepted: 6 November 2019

Published online: 11 November 2019

\section{References}

1. Younossi ZM, Koenig AB, Abdelatif D, Fazel Y, Henry L, Wymer M. Global epidemiology of nonalcoholic fatty liver disease-meta-analytic assessment of prevalence, incidence, and outcomes. Hepatology. 2016;64:73-84.

2. Anstee QM, McPherson S, Day CP. How big a problem is non-alcoholic fatty liver disease? BMJ. 2011;343:d3897.

3. Yki-Jarvinen $\mathrm{H}$. Non-alcoholic fatty liver disease as a cause and a consequence of metabolic syndrome. Lancet Diabetes Endocrinol. 2014;2: 901-10.

4. Chang Y, Jung HS, Yun KE, Cho J, Cho YK, Ryu S. Cohort study of nonalcoholic fatty liver disease, NAFLD fibrosis score, and the risk of incident diabetes in a Korean population. Am J Gastroenterol. 2013;108:1861-8.

5. Targher G, Byrne CD. Non-alcoholic fatty liver disease: an emerging driving force in chronic kidney disease. Nat Rev Nephrol. 2017;13:297-310.

6. Bellentani S, Dalle Grave R, Suppini A, Marchesini G, Fatty liver Italian N. Behavior therapy for nonalcoholic fatty liver disease: The need for a multidisciplinary approach. Hepatology. 2008;47:746-54.

7. Malhotra N, Beaton MD. Management of non-alcoholic fatty liver disease in 2015. World J Hepatol. 2015;7:2962-7.

8. Kuroda Y, Hara Y. Antimutagenic and anticarcinogenic activity of tea polyphenols. Mutat Res. 1999;436:69-97.

9. Stangl V, Lorenz M, Stangl K. The role of tea and tea flavonoids in cardiovascular health. Mol Nutr Food Res. 2006;50:218-28.

10. Suzuki Y, Miyoshi N, Isemura M. Health-promoting effects of green tea. Proc Jpn Acad Ser B Phys Biol Sci. 2012;88:88-101.

11. Mansour-Ghanaei F, Hadi A, Pourmasoumi M, Joukar F, Golpour S, Najafgholizadeh A. Green tea as a safe alternative approach for nonalcoholic fatty liver treatment: a systematic review and meta-analysis of clinical trials. Phytother Res. 2018;32:1876-84.

12. Song K, Du H, Zhang Q, Wang C, Guo Y, Wu H, et al. Serum immunoglobulin $\mathrm{M}$ concentration is positively related to metabolic syndrome in an adult population: Tianjin chronic low-grade systemic inflammation and health (TCLSIH) cohort study. PLoS One. 2014;9:e88701.

13. Yang YX, Wang GY. China food composition. 2rd ed. Beijing, China: Peking University Medical Press; 2009.

14. Fan JG, Jia JD, You Ming LI, Wang BY, Lun Gen LU, Shi JP, et al. Guidelines for the diagnosis and management of nonalcoholic fatty liver disease: update 2010. J Dig Dis. 2011:12:45-50.

15. Bassett DR Jr. International physical activity questionnaire: 12-country reliability and validity. Med Sci Sports Exerc. 2003;35:1396.

16. James PA, Oparil S, Carter BL, Cushman WC, Dennison-Himmelfarb C, Handler J, et al. 2014 evidence-based guideline for the management of high blood pressure in adults: report from the panel members appointed to the eighth joint National Committee (JNC 8). JAMA. 2014;311:507-20.

17. American DA. Diagnosis and classification of diabetes mellitus. Diabetes Care. 2014:37(Suppl 1):S81-90.

18. Joint committee for guideline r. 2016 Chinese guidelines for the management of dyslipidemia in adults. J Geriatr Cardiol. 2018;15:1-29.

19. Papavagelis C, Avgeraki E, Augoulea A, Stamatelopoulos K, Lambrinoudaki I, Yannakoulia M. Dietary patterns, Mediterranean diet and obesity in postmenopausal women. Maturitas. 2018;110:79-85.

20. Yang CQ, Shu L, Wang S, Wang JJ, Zhou Y, Xuan YJ, et al. Dietary patterns modulate the risk of non-alcoholic fatty liver disease in Chinese adults. Nutrients. 2015;7:4778-91. 
21. Adriano LS, Sampaio HA, Arruda SP, Portela CL, de Melo MLP, Carioca AA, et al. Healthy dietary pattern is inversely associated with non-alcoholic fatty liver disease in elderly. Br J Nutr. 2016;115:2189-95.

22. Kang JS, Lee WK, Yoon WK, Kim N, Park SK, Park HK, et al. A combination of grape extract, green tea extract and L-carnitine improves high-fat dietinduced obesity, hyperlipidemia and non-alcoholic fatty liver disease in mice. Phytother Res. 2011;25:1789-95.

23. Koo SI, Noh SK. Green tea as inhibitor of the intestinal absorption of lipids: potential mechanism for its lipid-lowering effect. J Nutr Biochem. 2007;18:179-83.

24. Lee MS, Kim CT, Kim Y. Green tea (-)-epigallocatechin-3-gallate reduces body weight with regulation of multiple genes expression in adipose tissue of diet-induced obese mice. Ann Nutr Metab. 2009;54:151-7.

25. Kim JA. Mechanisms underlying beneficial health effects of tea catechins to improve insulin resistance and endothelial dysfunction. Endocr Metab Immune Disord Drug Targets. 2008:8:82-8.

26. Na HK, Surh YJ. Modulation of Nrf2-mediated antioxidant and detoxifying enzyme induction by the green tea polyphenol EGCG. Food Chem Toxicol. 2008:46:1271-8.

27. Younossi ZM. Non-alcoholic fatty liver disease - a global public health perspective. J Hepatol. 2019;70:531-44.

28. Sakata R, Nakamura T, Torimura T, Ueno T, Sata M. Green tea with highdensity catechins improves liver function and fat infiltration in non-alcoholic fatty liver disease (NAFLD) patients: a double-blind placebo-controlled study. Int J Mol Med. 2013;32:989-94.

29. Lee SA, Dai Q, Zheng W, Gao YT, Blair A, Tessari JD, et al. Association of serum concentration of organochlorine pesticides with dietary intake and other lifestyle factors among urban Chinese women. Environ Int. 2007;33:157-63.

30. Liu X, Xu W, Cai H, Gao YT, Li H, Ji BT, et al. Green tea consumption and risk of type 2 diabetes in Chinese adults: the Shanghai Women's health study and the Shanghai Men's health study. Int J Epidemiol. 2018.

31. Binukumar BK, Bal A, Kandimalla R, Sunkaria A, Gill KD. Mitochondrial energy metabolism impairment and liver dysfunction following chronic exposure to dichlorvos. Toxicology. 2010;270:77-84.

32. Rezg R, Mornagui B, El-Fazaa S, Gharbi N. Biochemical evaluation of hepatic damage in subchronic exposure to malathion in rats: effect on superoxide dismutase and catalase activities using native PAGE. C R Biol. 2008;331:655-62.

33. Saadeh S, Younossi ZM, Remer EM, Gramlich T, Ong JP, Hurley M, et al. The utility of radiological imaging in nonalcoholic fatty liver disease. Gastroenterology. 2002:123:745-50.

\section{Publisher's Note}

Springer Nature remains neutral with regard to jurisdictional claims in published maps and institutional affiliations.

Ready to submit your research? Choose BMC and benefit from:

- fast, convenient online submission

- thorough peer review by experienced researchers in your field

- rapid publication on acceptance

- support for research data, including large and complex data types

- gold Open Access which fosters wider collaboration and increased citations

- maximum visibility for your research: over $100 \mathrm{M}$ website views per year

At $\mathrm{BMC}$, research is always in progress.

Learn more biomedcentral.com/submissions 